\title{
Tanshinones suppress AURKA through up-regulation of miR-32 expression in non-small cell lung cancer
}

\author{
Zhong-Liang Ma ${ }^{1, *}$, Bing-Jie Zhang ${ }^{1, *}$, De-Tao Wang ${ }^{1}$, Xue Li ${ }^{1}$, Jia-Li Wei ${ }^{1}$, \\ Bo-Tao Zhao', Yan Jin'², Yan-Li Li ${ }^{1}$, You-Xin Jin ${ }^{1,3}$ \\ ${ }^{1}$ School of Life Sciences, Shanghai University, Shanghai 200444, China \\ ${ }^{2}$ Institute of Biomedicine and Biotechnology, Shenzhen Institutes of Advanced Technology, Chinese Academy of Sciences, \\ Shenzhen 518055, China \\ ${ }^{3}$ State Key Laboratory of Molecular Biology, Institute of Biochemistry and Cell Biology, Institutes for Biological Sciences, \\ Chinese Academy of Sciences, Shanghai 200031, China \\ "These authors have contributed equally to this work
}

Correspondence to:

You-Xin Jin, e-mail: jinyouxin@shu.edu.cn

Yan-Li Li, e-mail: liyanli@shu.edu.cn

Yan Jin, e-mail: yan.jin@siat.ac.cn

Keywords: tanshinone, AURKA, miRNA, non-small cell lung cancer

Received: January 26, $2015 \quad$ Accepted: May 04, $2015 \quad$ Published: May 14, 2015

\section{ABSTRACT}

Tanshinone is the liposoluble constituent of Salia miltiorrhiza, a root used in traditional herbal medicine which is known to possess certain health benefits. Although it is known that tanshinones, including tanshinone I (T1), tanshinone IIA (T2A), and cryptotanshinone (CT), can inhibit the growth of lung cancer cells in vitro, the mechanism under which they act is still unclear. AURKA, an oncogene, encodes a serine-threonine kinase which regulates mitotic processes in mammalian cells. Here, we reported that tanshinones mediate AURKA suppression partly through upregulating the expression of miR-32. We found that tanshinones could inhibit cell proliferation, promote apoptosis, and impede cell-cycle progression, thus performing an antineoplastic function in non-small cell lung cancer (NSCLC). Additionally, we demonstrated that tanshinones attained these effects in part by down-regulating AURKA, corroborating previous reports. Our results showed that in NSCLC, similar effects were obtained with knock-down of the AURKA gene by siRNA. We also verified that AURKA was the direct target of miR-32. Collectively, our results demonstrated that tanshinones could inhibit NSCLC by suppressing AURKA via up-regulating the expressions of miR-32 and other related miRNAs.

\section{INTRODUCTION}

In recent years, there have been several advances in the treatment of lung cancer, notably a diversification in therapeutic drugs, as well as mode of treatment. Among these, targeted therapies have taken the forefront based on such features as efficiency and a low rate of adverse reactions [1-4]. Tanshinones, including tanshinone I (T1), tanshinone IIA (T2A), and cryptotanshinone (CT), are the liposoluble constituents of Salia miltiorrhiza, a traditional herbal therapy which has favorable medicinal value [5]. Of particular interest is tanshinone's cytotoxic effect on tumor cells, which is thought to act primarily through inducting apoptosis and interdicting cell cycle progression, angiogenesis, cell invasion and metastasis [6-9]. Related studies have indicated that tanshinones might effectively target human lung cancer cells and could inhibit growth by induction of apoptosis $[6,10-12]$. Thus, it is reasonable to speculate that tanshinones would play an important role in the treatment of non-small cell lung cancer (NSCLC). Research shows that tanshinone molecules inhibit the growth of lung cancer cells in vitro [13] and that the mechanism may be via interruption of cell cycle progression and induction of cell apoptosis, resulting in down-regulation in the expression of cell-cycle related proteins, Aurora A and Cyclin B, as well as the apoptosis related protein $\mathrm{Bcl} 2$. 
Aurora $\mathrm{A}$ is a serine-threonine kinase encoded by the AURKA (aurora kinase A) gene, which is responsible for regulating mitotic processes in mammalian cells, including centrosome maturation, spindle assembly, and chromosome segregation. Various types of cancers exhibit amplification of AURKA and serious effects such as chromosomal instability, centrosomal amplification/aneuploidy, therapeutic resistance, cell-cycle progression and anti-apoptosis are induced by overexpression of AURKA. Synergistically, these events promote the progression of cancer. Hence, AURKA can be considered an oncogene and thus an important target for cancer therapy.

MicroRNAs (miRNAs) are a class of single stranded small non-coding RNA. They are about 18-23 nucleotides (nt) in length, encoded by an endogenous gene, and regulate gene expression at the post-transcriptional level. Importantly, altered expression of miRNAs is reported in a variety of human cancers and may be associated with cancer pathogenesis, tumor growth, and metastasis $[14,15]$. Because miRNAs play a regulatory role in the tumorigenesis process and can regulate the expression of tumor associated genes [15-17], we proposed that tanshinones may regulate the expression of AURKA via adjusting the expression of related miRNAs.

\section{RESULTS}

\section{Tanshinones inhibit cell proliferation, promote apoptosis and impede cell-cycle progression in NSCLC}

To verify the suppression role of tanshinones in NSCLC, we first measured its anti-proliferative effects in several NSCLC cell lines, such as H1299, A549, and SPCA-1. The results showed that tanshinones could inhibit the proliferation of NSCLC cells in a time- and dosedependent manner (Figure 1A, Supplementary Figure S1A-S1B) and that cell proliferation was significantly inhibited by tanshinones at concentrations of $2 \mu \mathrm{M} / 4 \mu \mathrm{M}$ for $\mathrm{T} 1,2 \mu \mathrm{M} / 4 \mu \mathrm{M}$ for $\mathrm{T} 2 \mathrm{~A}$, and $5 \mu \mathrm{M} / 7.5 \mu \mathrm{M}$ for $\mathrm{CT}$ $(P<0.005 / 0.001)$ in $\mathrm{H} 1299$ cells (Figure 1A). In addition, results also indicated that $\mathrm{T} 1$ was the most effective of the tanshinones tested and that DMSO, the solvent of tanshinones, had no effect on cell proliferation.

The H1299 cell line was chosen to test the effects of tanshinones on apoptosis, cell cycle, and cell migration. The percentages of apoptotic cells in the tanshinonetreated groups were much higher than in the control (Figure 1B). Following treatment with tanshinones, the proportion of cells at the G0/G1 phase increased more than $10 \%$ as compared with the control (Figure 1C). Almost no difference was observed in the ability of cell migration between the experimental groups and the control group (Supplementary Figure S2). These results suggested that tanshinones could significantly promote apoptosis (Figure 1B) and cause G0/G1 cell-cycle arrest (Figure 1C) in H1299 cells. Thus tanshinones could exhibit an important antineoplastic effect in NSCLC tumor cells via inhibition of cell proliferation, promotion of apoptosis, and retardation of cell-cycle progression.

\section{Tanshinones inhibit NSCLC by down-regulating the expression of AURKA}

Li et al. [13] found that, in NSCLC, the suppressive effect of tanshinones may be due partly to down-regulation
A

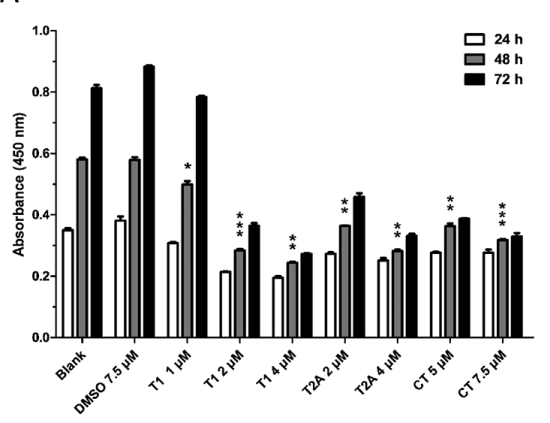

B
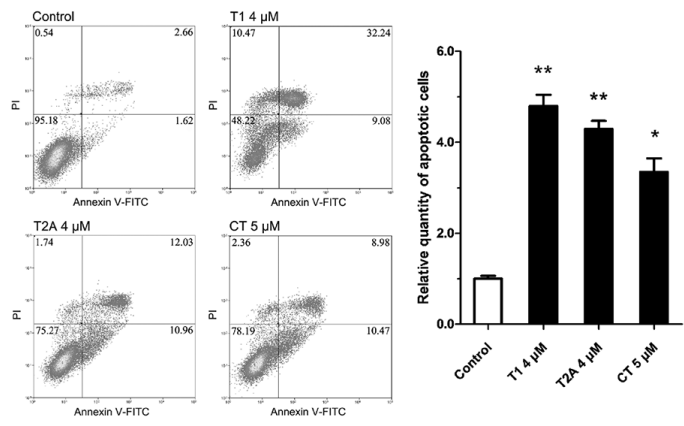

C
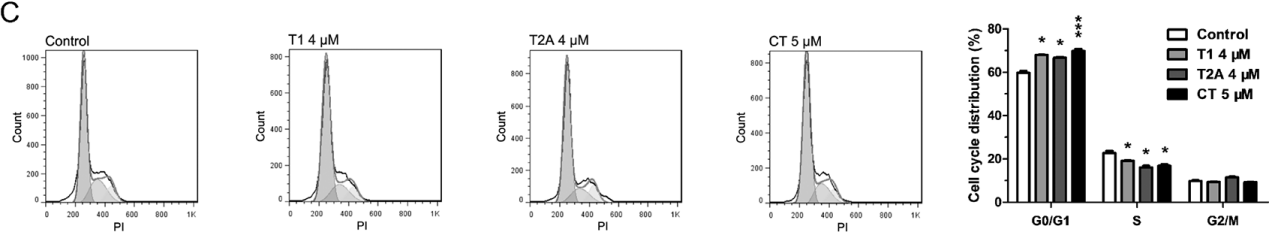

Figure 1: Tanshinone can suppress NSCLC. A. Cell vitality of H1299 cells treated with tanshinone or DMSO was determined by CCK- 8 cytotoxicity test. Results are represented as the mean \pm SEM of $\mathrm{OD}_{450 \mathrm{~nm}}$. Blank serves as control. In B-C. H1299 cells were respectively treated with T1 $4 \mu \mathrm{M}$, T2A $4 \mu \mathrm{M}$, CT $5 \mu \mathrm{M}$ or DMSO $5 \mu \mathrm{M}$ (Control) for $48 \mathrm{~h}$. B. Cell apoptosis situations of H1299 cells were detected by flow cytometry. C. Cell cycle distributions of $\mathrm{H} 1299$ cells were detected by flow cytometry. $* P<0.05, * * P<0.01$, $* * * P<0.001$, vs. control $(n=3)$. Representative of triplicate experiments was shown. 
of AURKA. To confirm this, we checked the variation of AURKA mRNA and protein after exposure to tanshinones (Figure 2A). Our data revealed that, in H1299 cells incubated for $48 \mathrm{~h}$ with $4 \mu \mathrm{M} \mathrm{T} 1,4 \mu \mathrm{M}$ T2A, or $5 \mu \mathrm{M} \mathrm{CT}$, the contents of AURKA mRNA and protein were much lower than the control group (DMSO $5 \mu \mathrm{M}$ for $48 \mathrm{~h}$ ) (Figure 2A). This result indicated that tanshinones could suppress the expression of AURKA. To further study the role of AURKA in NSCLC, we knocked down AURKA using siRNA and then surveyed the change in cell proliferation, apoptosis, and cell-cycle progression. After $24 \mathrm{~h}$ post-transfection with siAURKA/siNC, the content of AURKA mRNA decreased by almost $90 \%$ as compared to the control (Figure $2 \mathrm{~B}$ ). The Aurora A protein decreased about $60 \%$ after transfection for $24 \mathrm{~h}$ (Figure 2B). Results additionally showed that knocked-down of AURKA could suppress cell proliferation, accelerate apoptosis, and impede cell-cycle progression in an effect similar to that of tanshinones treatment (Figure 2C2E). Besides, we measured the endogenous expression level of AURKA in common NSCLC cell lines and found that the expression level of AURKA in H1299 cells was almost 7 times of in BEAS-2B, control cells (Supplementary Figure S3). In SPCA-1, the expression level of AURKA was about treble of in BEAS-2B. There approximately no difference between in A549 and in BEAS-2B. This result could explain why H1299 cells were much more sensitive to tanshinones than SPCA-1 and A549 cells (Figure 1A, Supplementary Figure S1A-S1B). Based on the above-mentioned results, it is reasonable to surmise that tanshinones restrain NSCLC in part by down-regulating the expression of AURKA.

A
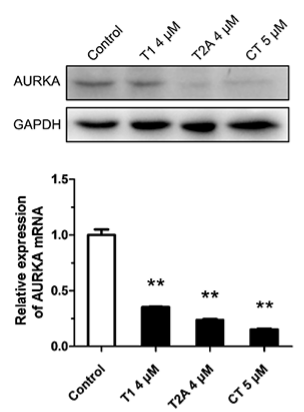
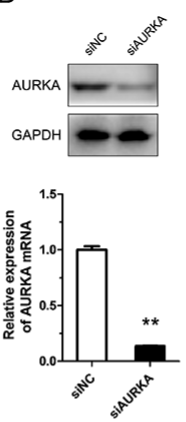

\section{Tanshinones suppress AURKA partly via up-regulating the expression of miR-32}

Although we know of the relationship between tanshinones and AURKA, the mechanism by which tanshinones regulate AURKA is still unclear. It has been well established that miRNAs regulate gene expression at the post-transcriptional level and that abnormal expression and regulation of miRNAs are critically involved in tumorigenesis and tumor progression. MiRNAs can regulate the expression of tumor associated genes and play a central role in the tumorigenesis process. Therefore, we propose that tanshinones may regulate the expression of AURKA by adjusting the expression of related miRNA. We searched for miRNAs that might target AURKA and select the most promising ones for further investigation by TargetScanHuman 6.2, StarBase v2.0, miRanda and http://microRNA.org (Supplementary Table 1). Although miR-34a was not part of the original list, we still tested it because of its explicit antitumor effect in lung cancer and its place as one of the miRNAs our group has researched [18]. Results from qRT-PCR assays showed that the expression levels of the miRNAs being tested (let-7b/c, miR-25, miR-32, miR-34a, miR-92a/b, miR-137, miR363 , and miR-367) were significantly up-regulated by tanshinones (T1, T2A, and CT) (Figure 3A, Supplementary Figure S4). We next focused on miR-32, as it has been reported that it may be an anti-oncogene in lung cancer but that its mechanism in NSCLC has yet to be brought to light [19-21]. We set out to unmask the target relationship
D

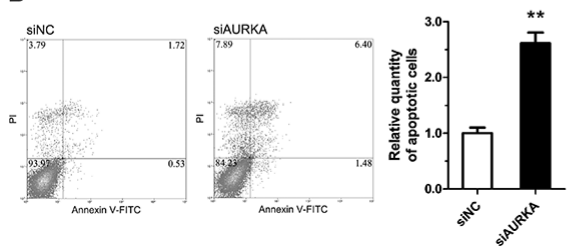

C

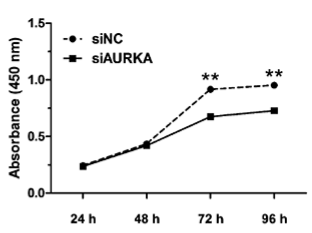

E
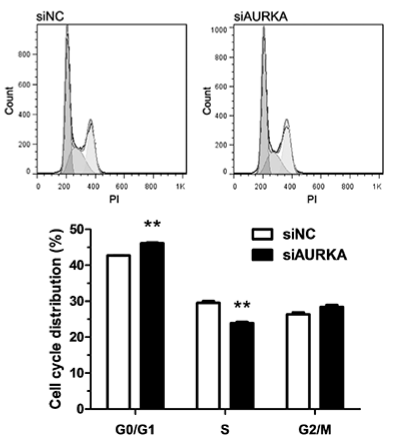

Figure 2: Tanshinone inhibits NSCLC by down-regulating the expression of AURKA. A. The variation of mRNA and protein of AURKA in $\mathrm{H} 1299$ cells respectively treated with $\mathrm{T} 14 \mu \mathrm{M}$, T2A $4 \mu \mathrm{M}$, CT $5 \mu \mathrm{M}$ or DMSO $5 \mu \mathrm{M}$ (Control) for 48 h were measured by qRT-PCR and Western blot. In B-E. H1299 cells were transfected with siAURKA or siNC (100nm) and incubated at $37^{\circ} \mathrm{C}$ for $24 \mathrm{~h}$ ( $96 \mathrm{~h}$ for CCK-8 cytotoxicity test ). B. The variation of mRNA and protein of AURKA in H1299 cells were measured by qRT-PCR and Western blot. C. Cell proliferation of H1299 cells were determined by CCK-8 cytotoxicity test. D. Cell apoptosis situations of H1299 cells were detected by flow cytometry. E. Cell cycle distributions of H1299 cells were detected by flow cytometry. ${ }^{* * P}<0.01$, vs. control $(n=3)$. Representative of triplicate experiments was shown. 
A
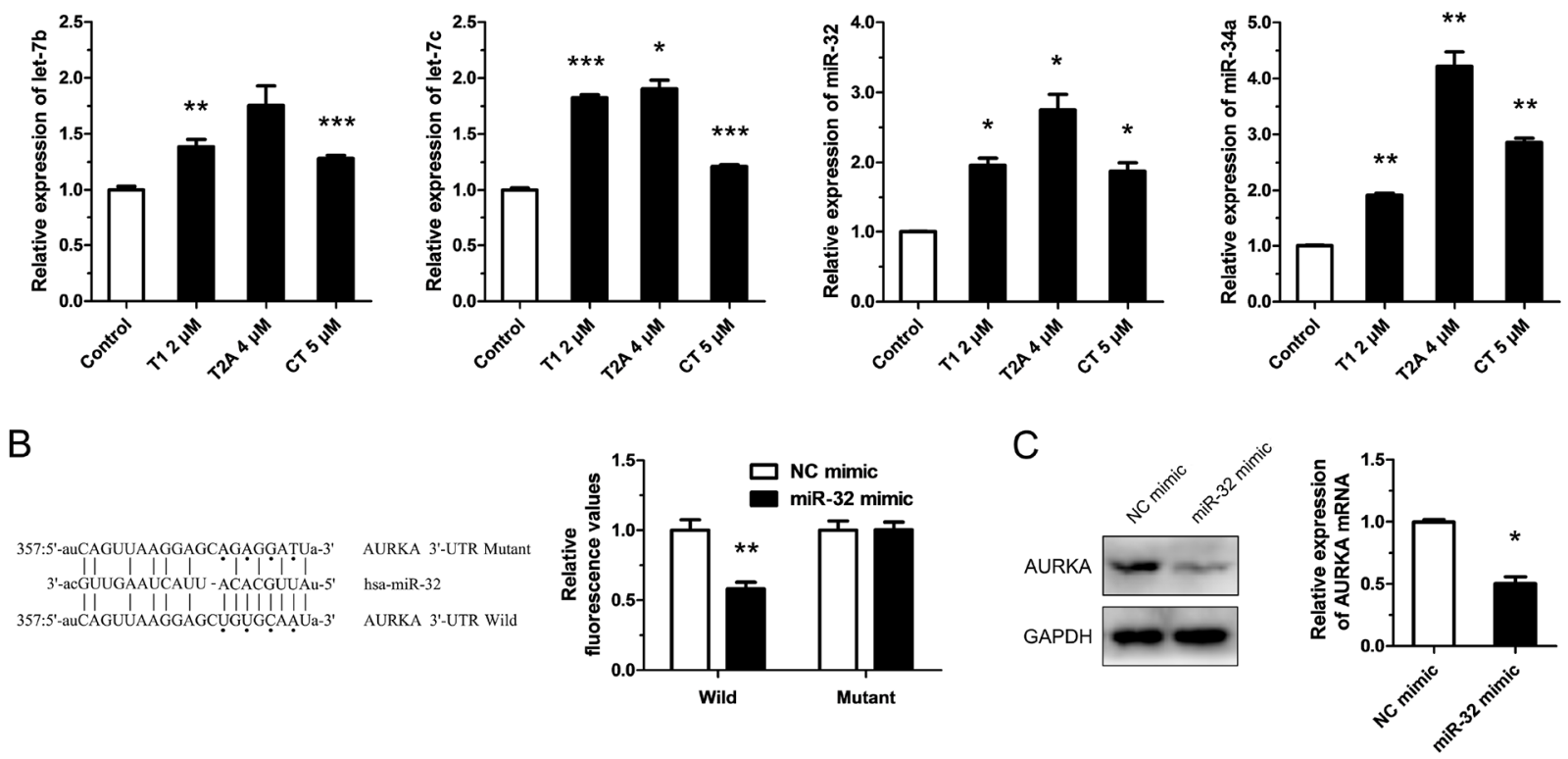

Figure 3: Tanshinone suppresses AURKA partly through up-regulating the expression of miR-32. A. Expression levels of miRNAs in H1299 cells respectively treated with T1 $4 \mu \mathrm{M}$, T2A $4 \mu \mathrm{M}$, CT $5 \mu \mathrm{M}$ or DMSO $5 \mu \mathrm{M}$ (Control) for $48 \mathrm{~h}$ were measured by qRTPCR. B. Dual luciferase reporter assay. Luciferase reporter constructs containing wild-type or mutated AURKA 3-UTR were cotransfected with miR-32 mimics or NC mimics into H1299 cells. Relative firefly luciferase expression was normalized to Renilla luciferase. C. The variation of mRNA and protein of AURKA in $\mathrm{H} 1299$ cells transfected with miR-32 mimics or NC mimics $(100 \mathrm{~nm})$ and incubated at $37^{\circ} \mathrm{C}$ for $24 \mathrm{~h}$ were measured by qRT-PCR and Western blot. ${ }^{*} P<0.05,{ }^{*} P<0.01,{ }^{* * *} P<0.001$, vs. control $(n=3)$. Representative of triplicate experiments was shown.

between miR-32 and AURKA. Data from the luciferase reporter assay revealed that there was a miR-32 binding site at 3'-UTR (357-379 bp) of AURKA (Figure 3B). Our results further showed that miR-32 mimic could downregulate the expressions of AURKA mRNA and protein (Figure 3C). These data suggested that miR-32 targeted AURKA and that tanshinones suppressed AURKA by regulating the expression levels of miR-32 and other interrelated miRNAs.

\section{miR-32 acts as a tumor suppressor gene in NSCLC}

Previous studies [19-21] indicated that miR-32 may be a tumor suppressor gene and our results confirmed that it could inhibit cell proliferation, promote apoptosis of tumor cells in NSCLC, but that it had no obvious effect on cell-cycle progression (Figure 4A-4C). We found that the antitumor effect of miR-32 was not conspicuous in H1299 cells, but we also measured the endogenous expression level of miR-32 in the other common NSCLC cell lines and found that the expression level of miR-32 in H1299 cells was much lower compared to BEAS-2B, control cells (Figure 4D). Jalava et al reported that androgen receptor (AR) could regulate miR32 expression by binding ARBS (AR-binding site) near the miR-32 genomic location [22]. Our results demonstrated tanshinones treatment can down-regulate the expression of $\mathrm{AR}$, and at the same time the expression of miR-32 is up-regulated in NSCLC (Supplementary Figure S5). When considering that one miRNA may target several genes and that a single gene could be the target of multiple miRNAs [23-27] along with negative feedback mechanisms that can exist in cells [28-30], it's reasonable to assume that one single miRNA may not have a remarkable effect on tumor suppression or promotion. According to these results as well as previous research, we concluded that miR-32 could play the role of tumor suppressor in NSCLC.

\section{DISCUSSION}

The value of tanshinones is well-known in both traditional Chinese medicine and modern medicine. As stated before, tanshinones have a cytotoxic effect on tumor cells via inducing apoptosis, arresting cellcycle progression, restraining angiogenesis, suppressing invasion and metastasis. In the present study, we proved that tanshinones (T1, T2A and CT) could significantly inhibit the proliferation of NSCLC cell lines in vitro via inducing apoptosis and retarding cell-cycle progression. These attributes might make it an ideal medicine for NSCLC treatment. Because there were still many details yet unknown about the primary mechanism of the suppression of NSCLC by tanshinones, it was necessary to validate whether tanshinones exhibited toxicity and if there was the presence of any by-effects on normal human cells. 
A

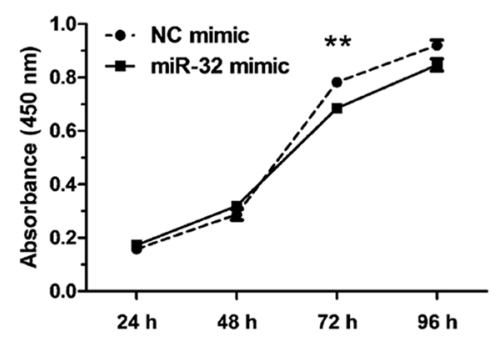

C

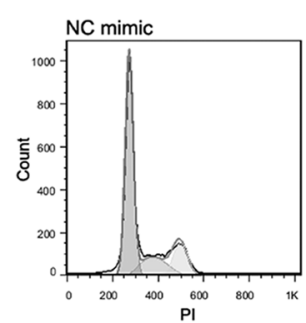

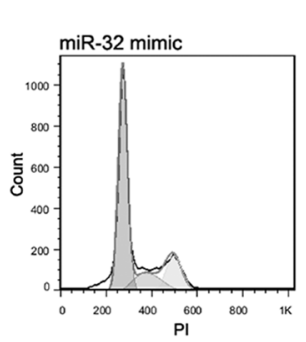

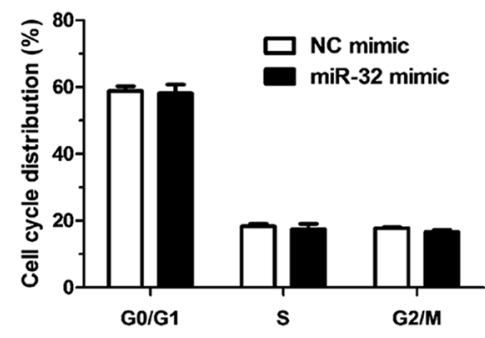

B
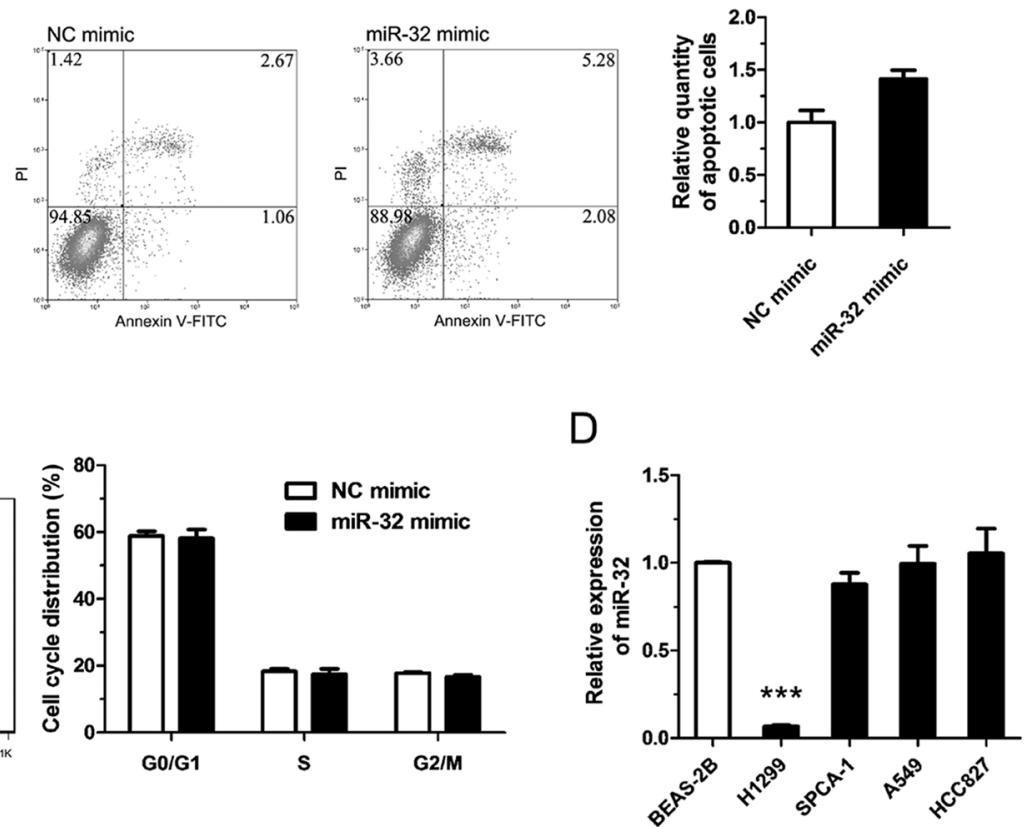

Figure 4: miR-32 plays a rule of tumor suppressor gene in NSCLC. In A-C, H1299 cells were transfected with NC mimics or miR-32 mimics $(100 \mathrm{~nm})$ and incubated at $37^{\circ} \mathrm{C}$ for $48 \mathrm{~h}(96 \mathrm{~h}$ for CCK-8 cytotoxicity test). A. Cell proliferation of H1299 cells were determined by CCK-8 cytotoxicity test. B. Cell apoptosis situations of H1299 cells were detected by flow cytometry. C. Cell cycle distributions of H1299 cells were detected by flow cytometry. D. The expression levels of miR-32 in lung cancer cell lines or pulmonary epithelial cell line (control) were measured by qRT-PCR. ${ }^{* *} P<0.01, * * * P<0.001$, vs. control $(n=3)$. Representative of triplicate experiments was shown.

AURKA is a member of a novel oncogenic family of mitotic serine/threonine kinases. Abundant evidence suggests a role for AURKA in centrosome maturation [31], spindle formation [32], and G2-M transition [33]. AURKA is frequently over expressed in different types of cancer [34-37] and suppression of AURKA expression and function reduces tumor growth [38-40]. Tumorigenesis is strongly related to abnormal amplification and expression of AURKA, which has led to the recognition of AURKA as an important molecular target for cancer therapy [41, 42]. Our studies showed that tanshinones down regulated the expression level of AURKA in vitro (Figure 2A, Supplementary Figure S6). These results suggest that AURKA is a novel and important molecular target for tanshinones.

The interrelation between tanshinones and AURKA has been preliminarily investigated, but the mechanism by which tanshinones up-regulate the expression of AURKA is still unclear. Herein, we validated the regulatory relationship between tanshinones and AURKA and defined the effects of AURKA in NSCLC. Above all, we found that tanshinones suppressed AURKA through regulating the expression levels of miR-32 and other interrelated miRNAs, and that AURKA is the direct target of miR-32. Nevertheless, the mechanism for regulation of miRNAs by tanshinones is almost utterly ignorant. In the near future, we hope to commence investigation on this topic.

In summary, our study identified a novel network delineating the method by which tanshinones suppress
NSCLC, first linking tanshinones with miRNAs and preliminary expounding the mechanism for this in NSCLC. Scientifically, this finding suggests tanshinones could efficiently be used as a targeted therapy in the treatment of NSCLC. Clinically, this study suggests AURKA as an ideal target for inhibiting cancer progression in NSCLC patients.

Based on our results thus far, we were able to summarize the mechanism by which tanshinones suppress NSCLC (Figure 5). Tanshinones can up-regulate the expression levels of tested miRNAs (Figure 3A, Supplementary Figure S4, Supplementary Figure S6), among these is let-7, a well-known tumor suppressor that can inhibit the development of lung cancer [43-45]. Our previous research revealed that miR-34a targets TGFßR2 which inhibits apoptosis in NSCLC [46] and in recent years, many studies have verified that miR-34a could inhibit tumor progression in lung cancer $[47,48]$. It was reported that miR-25, miR-32 and miR$92 \mathrm{a} / \mathrm{b}$ are in the same miRNA family [49] and based on this, they may play roles in a specific cancer. miR25 inhibition led to autophagic cell death by directly increasing ULK1 expression in breast cancer cell [50]. MiR-32 inhibits osteosarcoma cell proliferation and invasion by targeting Sox9 [20], however miR-92a/b as oncogene, promotes cell proliferation and invasion in cancer $[24,51]$. miR-25 family plays muti-roles in cancergenisis, we first report that $\mathrm{T} 1$ promotes miR-25 family and suppress the growth of NSCLC. 


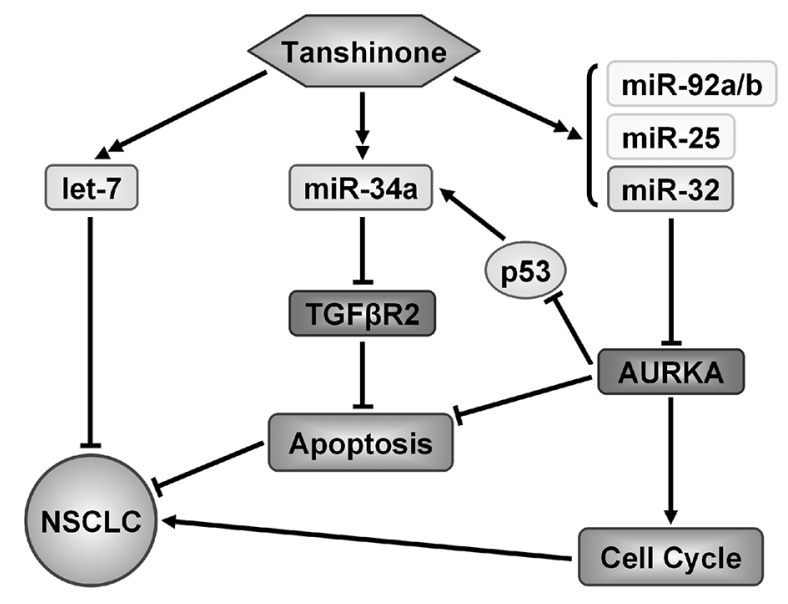

Figure 5: Model of identified mechanism how tanshinone suppresses NSCLC. Tanshinone played a role of tumor suppressor in NSCLC by up-regulating several miRNAs to suppress genes which related to the cancer process.

Our results demonstrated that miR-32 can suppress NSCLC by targeting AURKA (Figure 3B/3C, Figure 4) and it has been published that AURKA can suppress the effects of p53 [52, 53], an anti-oncogene which can up-regulate the expression level of miR-34a [54]. We also checked the the variation of AURKA/p53 mRNA and related miRNAs after exposure to tanshinones (Supplementary Figure S6). The result showed that, in SPCA-1 cells incubated for $48 \mathrm{~h}$ with $4 \mu \mathrm{M} \mathrm{T} 1,6 \mu \mathrm{M} \mathrm{T} 2 \mathrm{~A}$, the content of AURKA mRNA was much lower than the control group (DMSO $6 \mu \mathrm{M}$ for $48 \mathrm{~h}$ ) (Supplementary Figure S6). To the contrary, the contents of p53 mRNA and miRNAs were mucher higher than control group. This result indirectly proved that tanshinones could abolish the AURKA mediated p53 suppression and up-regulate the expressions of related miRNA. Comprehensively, tanshinones suppress NSCLC mainly through up-regulating anti-oncogene miRNAs to suppress their target genes that promote cancer progression, therefore giving tanshinones the ultimate effect of promoting apoptosis and arresting cell-cycle (Figure 5).

\section{MATERIALS AND METHODS}

\section{Chemicals and reagents}

Tanshinones (T1, T2A, and CT) were obtained from Nanjing University (Nanjing, China) and dissolved in dimethyl sulfoxide (DMSO). The U6 and relevant miRNAs primers (Supplementary Table 1) were designed and made by Invitrogen (Shanghai, China). The CellTiter $96{ }^{\circledR}$ AQueous One Solution Cell Proliferation Assay was purchased from Promega (Beijing, China) and the Annexin V-FITC Apoptosis Detection Kit was purchased from BD Biosciences (USA). The One Step PrimeScript $^{\circledR}$ miRNA cDNA Synthesis Kit and SYBR Premix Ex Taq II (both, Perfect Real Time) were purchased from Takara (Dalian, China). All chemical reagents were purchased from Sangon Biotech (Shanghai, China) or Sinopharm Chemical Reagent (Shanghai, China).

\section{Cell culture}

The human NSCLC cell line H1299 was obtained from ATCC. The human NSCLC cell lines A549, SPCA$1, \mathrm{HCC} 827$ and BEAS-2B cell line which isolated from normal human bronchial epithelium were obtained from the Cell Bank, China Academy of Sciences (Shanghai, China). The HEK-293T cell line was obtained from the Cell Bank as well. BEAS-2B cells were cultured in LHC9 medium (Gibco, Gaithersburg, USA). NSCLC cell lines were cultured in either RPMI 1640 or DMEM medium (Gibco). All the media were supplemented with $10 \%(\mathrm{v} / \mathrm{v})$ fetal bovine serum (FBS). Cells were cultured at $37^{\circ} \mathrm{C}$ in a $5 \% \mathrm{CO}_{2}$ atmosphere.

\section{Transfection}

H1299 cells were transiently transfected with $100 \mathrm{nM}$ of the chemically synthesized miR-32 mimic, negative control mimic (NC), miR-32 inhibitor, negative control inhibitor (NCi), siAURKA (sequence: 5'-AUGCCCUGUCUUACUGUCATT-3'), or negative control siRNA (siNC) (Ribobio, Guangzhou, China) using Lipofectamine 2000 (Invitrogen) according to the manufacturer's recommendations. After 24 to $48 \mathrm{~h}$ posttransfection, cells were used for subsequent experiments including assays for proliferation, western blot, apoptosis and cell cycle analysis.

\section{Cell proliferation analysis}

Cell proliferation was assessed by Cell Counting Kit-8 (CCK-8) assay kit (Dojindo, Japan). Cells treated with tanshinones (T1 at 1,2, and $4 \mu \mathrm{M}$; T2A at 2, 4, and $6 \mu \mathrm{M}$; CT at 5 and $7.5 \mu \mathrm{M})$, mimic, inhibitor, or siRNA 
were plated in a 96-well microplate (Corning Incorporated, Shanghai, China) and incubated at $37^{\circ} \mathrm{C}$ in a $5 \% \mathrm{CO}_{2}$ atmosphere. Data were obtained from the measurement of 4 replicate wells for each data point. After incubation for $24,48,72$ or $96 \mathrm{~h}, 10 \mu \mathrm{l}$ of CCK-8 solution was added to the appropriate wells and incubated for $60 \mathrm{~min}$. The absorbance was then measured by a multi-function enzyme-linked analyzer, FLx8 (BioTek, Shanghai, China), at the wavelength of $450 \mathrm{~nm}$ (OD, cell survival state).

\section{RNA isolation, reverse transcription and quantitative real-time PCR (qRT-PCR)}

Following the manufacturers' instructions, total RNA was isolated using Trizol Reagent (Sangon Biotech, Shanghai, China) and cDNA synthesis was performed with the PrimeScript ${ }^{\mathrm{TM}} 1$ st Strand cDNA Synthesis Kit or SYBR ${ }^{\circledR}$ PrimeScript ${ }^{\mathrm{TM}}$ miRNA RT -PCR Kit (TaKaRa). Quantitative RT-PCR analysis was performed using SYBR Green II (TaKaRa a) on a CFX96 ${ }^{\text {TM }}$ Real-time System (Bio-Rad, Shanghai, China) according to the manufacturer's protocol. The expression levels of miRNAs were normalized to the U6 expression level. The expression levels of mRNAs were normalized to the $18 \mathrm{~S}$ expression level.

\section{Cell apoptosis analysis}

Cell apoptosis was determined by the Annexin V-FITC/ propidium iodide (PI) apoptosis detection kit (BD Biosciences) following the manufacturer's protocol. Cells $\left(1 \times 10^{5}\right.$ cells $)$ were collected by trypsinization and centrifugation, then washed twice with phosphatebuffered saline (PBS). The cells were then resuspended in $100 \mu \mathrm{L} 1 \times$ binding buffer, to which $5 \mu \mathrm{L}$ of Annexin V-FITC and $5 \mu \mathrm{L}$ of PI were added and incubated at room temperature for $15 \mathrm{~min}$ in the dark, after which $400 \mu \mathrm{L} 1 \times$ binding buffer was added and mixed. Apoptotic cells were analyzed by a MoFlo XDP flow cytometry sorting system (Beckman Coulter, Mountain View, CA).

\section{Cell cycle analysis}

Treated cells $\left(1 \times 10^{5}\right.$ cells $)$ were harvested and fixed with $75 \%$ ethanol at $-20^{\circ} \mathrm{C}$ overnight, then washed twice with PBS. The cells were then resuspended in $250 \mu \mathrm{L}$ of RNase A buffer $(100 \mathrm{ng} / \mathrm{mL})$ and incubated at room temperature for $30 \mathrm{~min}$. After this incubation $2 \times \mathrm{PI}(100$ $\mathrm{ng} / \mathrm{mL}$ ) was added into the mixture and incubated for 15 min in the dark, followed by filtration with a 200 mesh filter membrane. Cell cycle was determined based on analysis on the MoFlo XDP flow cytometry sorting system (Becton Dickinson).

\section{Western blot analysis}

Total protein was extracted by RIPA lysis buffer (CWBIO, Beijing, China) and quantified using a modified
Bradford method. Equal amounts of protein samples were subjected to sodium dodecyl sulfate-polyacrylamide gel electrophoresis and transferred to a polyvinylidene fluoride membrane (Millipore Corporation, Billerica, USA). The membrane was then soaked in tris-buffered saline Tween-20 buffer (TBST) (20 mM Tris-HCl, pH 8.0, $150 \mathrm{mM} \mathrm{NaCl}$, $0.05 \%$ Tween-20) with $5 \%$ bovine serum albumin (BSA), for $1 \mathrm{~h}$ at room temperature with gentle shaking and subsequently incubated with specific antibody against AURKA (1:1000, Cell Signaling Technology, Danvers, USA) or GAPDH (1:1000, ABclonal Technology, Wuhan, China) antibody for $2 \mathrm{~h}$ at room temperature or overnight at $4^{\circ} \mathrm{C}$. Afterwards, the membrane was washed and incubated with horseradish peroxidase (HRP)-conjugated secondary antibody (1:10000, Signalway Antibody, Nanjing, China) for $1 \mathrm{~h}$. Finally, protein bands were detected by a chemiluminescent HRP substrate (Millipore Corporation), quantitated by densitometric analysis using the Image Lab analysis software (Bio-Rad), and expressed as percentage of control after normalization to GAPDH.

\section{Dual luciferase reporter assay}

The wild type human AURKA 3'-UTR firefly luciferase construct (pGL3-AURKA-3'-UTR-wt) was generated by inserting a $719 \mathrm{bp}$ fragment of human AURKA 3'-UTR into the EcoR V/EcoR I sites of the pGL3 miReport vector. The pGL3-AURKA-3'-UTRmut construct was generated by mutating the putative miR-32-binding site on pGL3-AURKA-3'-UTR-wt. As a control for transfection efficiency, plasmid expressing the renilla luciferase gene (pRL) was cotransfected into each transfection experiment. Cells were harvested $48 \mathrm{~h}$ after transfection and luciferase activity was assayed by an Orion II Microplate Illuminometer (Titertek-Berthold, South San Francisco, USA). Relative activities were expressed as the fold-change in luciferase activity after normalization to renilla luciferase activity. All constructs were sequenced to verify integrity. All the primers used in this paper are listed in Supplementary Table 2.

\section{Statistical analysis}

Results were represented as the mean \pm SEM and difference between two experimental groups was evaluated using student's $t$-test with statistical significance defined as $P<0.05$.

\section{ACKNOWLEDGMENTS}

This work was supported in part by grants from National Natural Science Foundation of China (31170750, 31100570), the National Basic Research Program of China (2011CBA01105) and Shanghai University and a foundation from State Key Laboratory of Cell Biology, Institute of Biochemistry and Cell 
Biology, Shanghai Institutes for Life Sciences, Chinese Academy of Sciences.

\section{CONFLICTS OF INTEREST}

The authors declare no competing financial interests.

\section{REFERENCES}

1. Fujioka N, Bitterman PB. Molecular targeted therapy in lung cancer. Minn Med. 2012; 95:38-41.

2. Malgieri S, Feliciano S, Bosso D, Federico P, Palmieri G, De Placido S, Di Lorenzo G, Buonerba C. Non-small cell lung cancer: from targeted therapy to tailored therapy. Expert Opin Pharmacother. 2012; 13:1817-1819.

3. Petrosyan F, Daw H, Haddad A, Spiro T. Targeted therapy for lung cancer. Anticancer Drugs. 2012; 23:1016-1021.

4. Wu K, House L, Liu W, Cho WC. Personalized targeted therapy for lung cancer. Int J Mol Sci. 2012; 13:11471-11496.

5. Zhou L, Zuo Z, Chow MS. Danshen: an overview of its chemistry, pharmacology, pharmacokinetics, and clinical use. J Clin Pharmacol. 2005; 45:1345-1359.

6. Pan Y, Bi HC, Zhong GP, Chen X, Zuo Z, Zhao LZ, Gu LQ, Liu PQ, Huang ZY, Zhou SF, Huang M. Pharmacokinetic characterization of hydroxylpropyl-beta-cyclodextrin-included complex of cryptotanshinone, an investigational cardiovascular drug purified from Danshen (Salvia miltiorrhiza). Xenobiotica. 2008; 38:382-398.

7. Chen W, Luo Y, Liu L, Zhou H, Xu B, Han X, Shen T, Liu Z, Lu Y, Huang S. Cryptotanshinone inhibits cancer cell proliferation by suppressing Mammalian target of rapamycin-mediated cyclin $\mathrm{D} 1$ expression and $\mathrm{Rb}$ phosphorylation. Cancer Prev Res (Phila). 2010; 3:1015-1025.

8. Cheng CY, Su CC. Tanshinone IIA inhibits Hep-J5 cells by increasing calreticulin, caspase 12 and GADD153 protein expression. Int J Mol Med. 2010; 26:379-385.

9. Tang C, Xue HL, Huang HB, Wang XG. Tanshinone IIA inhibits constitutive STAT3 activation, suppresses proliferation, and induces apoptosis in rat $\mathrm{C} 6$ glioma cells. Neurosci Lett. 2010; 470:126-129.

10. Chiu TL, Su CC. Tanshinone IIA induces apoptosis in human lung cancer A549 cells through the induction of reactive oxygen species and decreasing the mitochondrial membrane potential. Int J Mol Med. 2010; 25:231-236.

11. Lee CY, Sher HF, Chen HW, Liu CC, Chen CH, Lin CS, Yang PC, Tsay HS, Chen JJ. Anticancer effects of tanshinone I in human non-small cell lung cancer. Mol Cancer Ther. 2008; 7:3527-3538.

12. Bi HC, Law FC, Zhong GP, Xu CS, Pan Y, Ding L, Chen X, Zhao LZ, Xu Q, Huang M. Study of tanshinone IIA tissue distribution in rat by liquid chromatography-tandem mass spectrometry method. Biomed Chromatogr. 2007; 21:473-479.

13. Li Y, Gong Y, Li L, Abdolmaleky HM, Zhou JR. Bioactive tanshinone I inhibits the growth of lung cancer in part via downregulation of Aurora A function. Mol Carcinog. 2013; 52:535-543.

14. Voortman J, Goto A, Mendiboure J, Sohn JJ, Schetter AJ, Saito M, Dunant A, Pham TC, Petrini I, Lee A, Khan MA, Hainaut P, Pignon JP, Brambilla E, Popper HH, Filipits M, et al. MicroRNA expression and clinical outcomes in patients treated with adjuvant chemotherapy after complete resection of non-small cell lung carcinoma. Cancer Res. 2010; 70:8288-8298.

15. Bartels CL, Tsongalis GJ. [MicroRNAs: novel biomarkers for human cancer]Ann Biol Clin. Paris: 2010; 68:263-272.

16. Liu J, Zheng M, Tang YL, Liang XH, Yang Q. MicroRNAs, an active and versatile group in cancers. Int J Oral Sci. 2011; 3:165-175.

17. Lu J, Getz G, Miska EA, Alvarez-Saavedra E, Lamb J, Peck D, Sweet-Cordero A, Ebert BL, Mak RH, Ferrando AA, Downing JR, Jacks T, Horvitz HR, Golub TR. MicroRNA expression profiles classify human cancers. Nature. 2005; 435:834-838.

18. Ma ZL, Hou PP, Li YL, Wang DT, Yuan TW, Wei JL, Zhao BT, Lou JT, Zhao XT, Jin Y, Jin YX. MicroRNA34a inhibits the proliferation and promotes the apoptosis of non-small cell lung cancer H1299 cell line by targeting TGFbetaR2. Tumour biology: the journal of the International Society for Oncodevelopmental Biology and Medicine. 2015; 36:2481-2490.

19. Dacic S, Kelly L, Shuai Y, Nikiforova MN. miRNA expression profiling of lung adenocarcinomas: correlation with mutational status. Mod Pathol. 2010; 23:1577-1582.

20. Xu JQ, Zhang WB, Wan R, Yang YQ. MicroRNA-32 inhibits osteosarcoma cell proliferation and invasion by targeting Sox9. Tumour biology : the journal of the International Society for Oncodevelopmental Biology and Medicine. 2014; 35:9847-9853.

21. Zhang J, Kuai X, Song M, Chen X, Yu Z, Zhang H, Mao Z. microRNA-32 inhibits the proliferation and invasion of the SGC-901 gastric cancer cell line. Oncol Lett. 2014; 7:270-274.

22. Jalava SE, Urbanucci A, Latonen L, Waltering KK, Sahu B, Janne OA, Seppala J, Lahdesmaki H, Tammela TL, Visakorpi T. Androgen-regulated miR-32 targets BTG2 and is overexpressed in castration-resistant prostate cancer. Oncogene. 2012; 31:4460-4471.

23. Mattiske S, Suetani RJ, Neilsen PM, Callen DF. The oncogenic role of miR-155 in breast cancer. Cancer Epidemiol Biomarkers Prev. 2012; 21:1236-1243.

24. Li Y, Li L, Guan Y, Liu X, Meng Q, Guo Q. MiR-92b regulates the cell growth, cisplatin chemosensitivity of A549 non small cell lung cancer cell line and target PTEN. Biochem Biophys Res Commun. 2013; 440:604-610. 
25. Xu LF, Wu ZP, Chen Y, Zhu QS, Hamidi S, Navab R. MicroRNA-21 (miR-21) regulates cellular proliferation, invasion, migration, and apoptosis by targeting PTEN, RECK and Bcl-2 in lung squamous carcinoma, Gejiu City, China. PLoS One. 2014; 9:e103698.

26. Jiang J, Zhang Y, Yu C, Li Z, Pan Y, Sun C. MicroRNA-492 expression promotes the progression of hepatic cancer by targeting PTEN. Cancer Cell Int. 2014; 14:95.

27. Geng L, Sun B, Gao B, Wang Z, Quan C, Wei F, Fang XD. MicroRNA-103 promotes colorectal cancer by targeting tumor suppressor DICER and PTEN. Int J Mol Sci. 2014; 15:8458-8472.

28. Park S, Zhao D, Hatanpaa KJ, Mickey BE, Saha D, Boothman DA, Story MD, Wong ET, Burma S, Georgescu MM, Rangnekar VM, Chauncey SS, Habib AA. RIP1 activates PI3K-Akt via a dual mechanism involving NF-kappaB-mediated inhibition of the mTOR-S6K-IRS1 negative feedback loop and down-regulation of PTEN. Cancer Res. 2009; 69:4107-4111.

29. Gong L, Wu Z, Guo L, Li L, Zhao R, Zhu D, Zhou Q. Metastasis suppressor Nm23-H1 inhibits STAT3 signaling via a negative feedback mechanism. Biochem Biophys Res Commun. 2013; 434:541-546.

30. Halappanavar S, Nikota J, Wu D, Williams A, Yauk CL, Stampfli M. IL-1 receptor regulates microRNA-135b expression in a negative feedback mechanism during cigarette smoke-induced inflammation. J Immunol. 2013; 190:3679-3686.

31. Marumoto T, Zhang D, Saya H. Aurora-A - a guardian of poles. Nat Rev Cancer. 2005; 5:42-50.

32. Hirota T, Kunitoku N, Sasayama T, Marumoto T, Zhang D, Nitta M, Hatakeyama K, Saya H. Aurora-A and an interacting activator, the LIM protein Ajuba, are required for mitotic commitment in human cells. Cell. 2003; 114:585-598.

33. Kaestner P, Stolz A, Bastians H. Determinants for the efficiency of anticancer drugs targeting either Aurora-A or Aurora-B kinases in human colon carcinoma cells. Mol Cancer Ther. 2009; 8:2046-2056.

34. Comperat E, Bieche I, Dargere D, Laurendeau I, Vieillefond A, Benoit G, Vidaud M, Camparo P, Capron F, Verret C, Cussenot O, Bedossa P, Paradis V. Gene expression study of Aurora-A reveals implication during bladder carcinogenesis and increasing values in invasive urothelial cancer. Urology. 2008; 72:873-877.

35. Lee EC, Frolov A, Li R, Ayala G, Greenberg NM. Targeting Aurora kinases for the treatment of prostate cancer. Cancer Res. 2006; 66:4996-5002.

36. Matarasso N, Bar-Shira A, Rozovski U, Rosner S, Orr-Urtreger A. Functional analysis of the Aurora Kinase A Ile31 allelic variant in human prostate. Neoplasia. 2007; 9:707-715.

37. Kumano M, Miyake H, Terakawa T, Furukawa J, Fujisawa M. Suppressed tumour growth and enhanced chemosensitivity by RNA interference targeting Aurora-A in the PC3 human prostate cancer model. BJU Int. 2010; 106:121-127.

38. Addepalli MK, Ray KB, Kumar B, Ramnath RL, Chile S, Rao H. RNAi-mediated knockdown of AURKB and EGFR shows enhanced therapeutic efficacy in prostate tumor regression. Gene Ther. 2010; 17:352-359.

39. Li Y, Zhang ZF, Chen J, Huang D, Ding Y, Tan MH, Qian CN, Resau JH, Kim H, Teh BT. VX680/MK-0457, a potent and selective Aurora kinase inhibitor, targets both tumor and endothelial cells in clear cell renal cell carcinoma. Am J Transl Res. 2010; 2:296-308.

40. Wang X, Dong L, Xie J, Tong T, Zhan Q. Stable knockdown of Aurora-A by vector-based RNA interference in human esophageal squamous cell carcinoma cell line inhibits tumor cell proliferation, invasion and enhances apoptosis. Cancer Biol Ther. 2009; 8:1852-1859.

41. Dar AA, Goff LW, Majid S, Berlin J, El-Rifai W. Aurora kinase inhibitors-rising stars in cancer therapeutics? Mol Cancer Ther. 2010; 9:268-278.

42. Gorgun G, Calabrese E, Hideshima T, Ecsedy J, Perrone G, Mani M, Ikeda H, Bianchi G, Hu Y, Cirstea D, Santo L, Tai YT, Nahar S, Zheng M, Bandi M, Carrasco RD, et al. A novel Aurora-A kinase inhibitor MLN8237 induces cytotoxicity and cell-cycle arrest in multiple myeloma. Blood. 2010; 115:5202-5213.

43. Barh D, Malhotra R, Ravi B, Sindhurani P. MicroRNA let-7: an emerging next-generation cancer therapeutic. Curr Oncol. 2010; 17:70-80.

44. Esquela-Kerscher A, Trang P, Wiggins JF, Patrawala L, Cheng A, Ford L, Weidhaas JB, Brown D, Bader AG, Slack FJ. The let-7 microRNA reduces tumor growth in mouse models of lung cancer. Cell Cycle. 2008; 7:759-764.

45. Osada H, Takahashi T. let-7 and miR-17-92: small-sized major players in lung cancer development. Cancer Sci. 2011; 102:9-17.

46. Ma ZL, Hou PP, Li YL, Wang DT, Yuan TW, Wei JL, Zhao BT, Lou JT, Zhao XT, Jin Y, Jin YX. MicroRNA$34 \mathrm{a}$ inhibits the proliferation and promotes the apoptosis of non-small cell lung cancer H1299 cell line by targeting TGFbetaR2. Tumour Biol. 2015; 36:2481-90.

47. Bandi N, Vassella E. miR-34a and miR-15a/16 are co-regulated in non-small cell lung cancer and control cell cycle progression in a synergistic and Rb-dependent manner. Mol Cancer. 2011; 10:55.

48. Shi Y, Liu C, Liu X, Tang DG, Wang J. The microRNA miR-34a inhibits non-small cell lung cancer (NSCLC) growth and the CD44hi stem-like NSCLC cells. PLoS One. 2014; 9:e90022.

49. Wahlquist C, Jeong D, Rojas-Munoz A, Kho C, Lee A, Mitsuyama S, van Mil A, Park WJ, Sluijter JP, Doevendans PA, Hajjar RJ, Mercola M. Inhibition of 
miR-25 improves cardiac contractility in the failing heart. Nature. 2014; 508:531-535.

50. Wang Z, Wang N, Liu P, Chen Q, Situ H, Xie T, Zhang J, Peng C, Lin Y, Chen J. MicroRNA-25 regulates chemoresistance-associated autophagy in breast cancer cells, a process modulated by the natural autophagy inducer isoliquiritigenin. Oncotarget. 2014; 5:7013-26.

51. Zhou C, Shen L, Mao L, Wang B, Li Y, Yu H. miR-92a is upregulated in cervical cancer and promotes cell proliferation and invasion by targeting FBXW7. Biochemical and biophysical research communications. 2015; 458:63-69.
52. Katayama H, Sasai K, Kawai H, Yuan ZM, Bondaruk J, Suzuki F, Fujii S, Arlinghaus RB, Czerniak BA, Sen S. Phosphorylation by aurora kinase A induces Mdm2-mediated destabilization and inhibition of p53. Nat Genet. 2004; 36:55-62.

53. Liu Q, Kaneko S, Yang L, Feldman RI, Nicosia SV, Chen J, Cheng JQ. Aurora-A abrogation of p53 DNA binding and transactivation activity by phosphorylation of serine 215 . J Biol Chem. 2004; 279:52175-52182.

54. Li J, Wang K, Chen X, Meng H, Song M, Wang Y, Xu X, Bai Y. Transcriptional activation of microRNA-34a by NF-kappa B in human esophageal cancer cells. BMC Mol Biol. 2012; 13:4. 